\title{
Erratum: Chain of Dirac spectrum loops of nodes in crossed magnetic and electric fields [Phys. Rev. B 97, 115204 (2018)]
}

\author{
V. I. Gavrilenko, A. A. Perov, A. P. Protogenov, R. V. Turkevich, and E. V. Chulkov
}

(Received 12 April 2018; published 24 April 2018)

DOI: 10.1103/PhysRevB.97.159901

In our paper, there are some minor mistakes which should be corrected. The two sentences before "When constructing the Landau damping regions, we used the following dimensionless variables" (p. 4, right column) should be replaced by the following ones: In the considered model, we found Landau damping regions for left-hand polarized waves, which take place for $n^{\prime}=n \pm 1$. The Landau damping regions for $n^{\prime}=n+1$ are plotted in Fig. 2.

The caption for Fig. 2 should be supplemented by the following sentence: To avoid inconvenience, we do not present here the Landau damping regions that correspond to the transitions $n \rightarrow n-1$ because there is no collisionless absorption related to these transitions in the region $0 \leqslant \Omega \leqslant 1, q \ll 1$.

The Acknowledgments should be changed as follows: We would like to thank M. D. Tokman for valuable remarks. The parts of this work related to the results of Secs. I-III were supported by the Russian Science Foundation through Grant No. 18-12-00169 (A.P.P.). The remaining part in Sec. IV was supported by Project No. 3.2637.2017/4.6 (A.A.P.) of the Ministry of Education and Science of the Russian Federation. The entire work was supported, in part, by the Basque Country University, Grant No. IT-756-13 (E.V.C.). One of the authors (A.P.P.) is grateful to the International Physics Center in San Sebastian for its hospitality. 